\title{
Obesidade e síndrome metabólica na infância e adolescência
}

\author{
Obesity and metabolic syndrome in infancy \\ and adolescence
}

Cecília Lacroix de OLIVEIRA ${ }^{1}$

Marco Túlio de MELLO²

Isa de Pádua CINTRA ${ }^{3}$

Mauro FISBERG ${ }^{3}$

\section{R E S U M O}

Nas últimas décadas a prevalência da obesidade vem apresentando um aumento em vários países ao redor do mundo. Este fato é preocupante, já que o excesso de gordura corporal, principalmente a abdominal, está diretamente relacionado com alterações do perfil lipídico, com o aumento da pressão arterial e a hiperinsulinemia, considerados fatores de risco para o desenvolvimento de doenças crônicas, como o diabetes melito tipo 2 e as doenças cardiovasculares. Níveis elevados de leptina e de ácido úrico e a alteração dos fatores fibrinolíticos também têm sido observados em indivíduos obesos. O conjunto destas alterações tem sido descrito como "síndrome metabólica" ou "síndrome da resistência à insulina", já que a hiperinsulinemia tem um papel importante no desenvolvimento dos outros componentes da síndrome metabólica. Entretanto, questiona-se se estas alterações já estão presentes em crianças e adolescentes obesos. Este artigo descreve a fisiopatologia dos componentes da síndrome metabólica e esclarece como este processo ocorre na faixa etária mais jovem.

Termos de indexação: obesidade, criança, adolescente, resistência à insulina, hiperlipidemia, hipertensão, síndrome metabólica.

\footnotetext{
1 Pós-graduanda, Centro de Apoio e Atendimento ao Adolescente, Departamento de Pediatria, Universidade Federal de São Paulo/Escola Paulista de Medicina. Rua Loefgreen, 1647, 04040-032, São Paulo, SP, Brasil. Correspondência para/Correspondence to: C.L. OLIVEIRA. E-mail: cecilacroix@yahoo.com.br

2 Departamento de Psicobiologia, Universidade Federal de São Paulo/Escola Paulista de Medicina.

${ }^{3}$ Centro de Apoio e Atendimento ao Adolescente, Departamento de Pediatria, Universidade Federal de São Paulo/Escola Paulista de Medicina.
} 


\section{A B S T R A C T}

The prevalence of obesity is increasing all over the world in the last decades. This is a concerning fact since the excess of body fat, especially abdominal fat, is directly related to the lipidic profile that is altered, to the increase of arterial pressure and hyperinsulinemia. All of these are considered risk factors to the onset of chronic-degenerative diseases such as type 2 diabetes and cardiovascular disease. High levels of leptin and the alterations of fibrinolytic factors have also been observed in obese individuals. These modifications are described as "metabolic syndrome" or "insulin resistance syndrome", since hyperinsulinemia has an important role in the development of other components of the metabolic syndrome. However, the question is if these alterations are already present in these children and adolescents. This paper describes the physiopathology of the metabolic syndrome components and aims at clarifying how this process takes place in youth.

Index terms: obesity, child, adolescent, insulin resistence, hyperlipidemia, hypertension, metabolic syndrome.

\section{N T R O D U Ç Ã O}

A obesidade está sendo considerada uma doença crônica e epidêmica, pois vem apresentando um rápido aumento em sua prevalência nas últimas décadas, tanto em países desenvolvidos como nos em desenvolvimento, e está relacionada com uma alta taxa de morbidade e mortalidade.

No Brasil, verifica-se um processo de transição nutricional nas últimas décadas. Comparando-se os dados do Estudo Nacional da Despesa Familiar (ENDEF), realizado em 1974/ 1975, com os dados da Pesquisa sobre Padrões de Vida (PPV), realizada em 1996/1997, somente nas regiões Sudeste e Nordeste verificou-se um aumento na prevalência de sobrepeso e obesidade de $4,1 \%$ para $13,9 \%$ em crianças e adolescentes de 6 a 18 anos ${ }^{1}$.

Estudos realizados em algumas cidades brasileiras mostram que o sobrepeso e a obesidade já atingem mais de $20 \%$ das crianças e adolescentes, como em Recife, alcançando 35\% dos escolares avaliados ${ }^{2}$.

É claro que as tendências da obesidade não são limitadas a um determinado grupo étnico ou região. Tem se verificado um aumento constante desta doença na América do Norte, América Latina, África do Sul, Malásia e nações do Pacífico.
Estudos epidemiológicos vêm mostrando uma associação do crescimento expressivo na incidência de doenças crônicas, como o diabetes melito tipo 2 e as doenças coronarianas, com a diminuição do tempo de vida livre de doenças, à medida que ocorre um aumento do IMC da população ${ }^{3}$.

Neste sentido, um aspecto que deve ser levado em consideração é a presença de lesões precoces de aterosclerose já nas primeiras décadas de vida. A presença de alterações metabólicas (resistência à insulina, dislipidemia, hipertensão, alterações trombogênicas, hiperuricemia) na infância e adolescência pode contribuir para o desenvolvimento deste processo, já que estudos longitudinais clássicos mostram uma forte associação entre o excesso de peso nas primeiras décadas de vida e a alta taxa de morbimortalidade na vida adulta por doenças cardiovasculares. Must et al. ${ }^{4}$ analisaram adolescentes do estudo de Harvard Growth, que foram acompanhados durante 55 anos, e verificaram que $52 \%$ dos indivíduos que apresentavam excesso de peso quando adolescentes permaneceram neste estado nutricional 55 anos após e o risco relativo para todas as causas de doenças coronarianas foi aproximadamente duas vezes maior nestes indivíduos.

Descrever as alterações metabólicas decorrentes da obesidade e analisá-las em crianças e adolescentes obesos é o objetivo deste artigo. 


\section{Morbidade e mortalidade associadas à obesidade}

Além do risco aumentado da criança e do adolescente obeso permanecer neste estado quando adultos se comparados aos indivíduos eutróficos, estudos longitudinais sugerem que o tempo de duração da obesidade está diretamente associado à morbi-mortalidade por doenças cardiovasculares (DCV). Srinivasan et al. ${ }^{5}$, verificaram em um estudo longitudinal que dos 191 adolescentes obesos, 110 (58\%) permaneceram neste estado na fase adulta (12 a 14 anos mais tarde) e tiveram uma prevalência de hipertensão 8,5 vezes maior e valores de LDL-C e de triglicérides de 3,1 e 8,3 vezes maior, respectivamente, em relação aos que não apresentaram sobrepeso.

A presença de pelo menos um fator de risco para DCV (hipertensão, dislipidemia ou hiperinsulinemia) tem sido observada em $60 \%$ das crianças e adolescentes com excesso de peso, sendo que $20 \%$ apresentam dois ou mais fatores de risco ${ }^{6}$. O Bogalusa Heart Study, realizado com 9167 indivíduos de 5 a 17 anos de idade, entre 1973 e 1994, com o objetivo de avaliar fatores de risco para doença cardiovascular nas primeiras décadas de vida, constatou que, entre os que apresentavam obesidade (813), 58\% tinham pelo menos um fator de risco (dislipidemia, hiperinsulinemia ou hipertensão arterial). As crianças e adolescentes obesos apresentavam também 2,4 vezes e 7,1 vezes maior chance de ter níveis de colesterol total e triglicérides, respectivamente, mais elevados do que os eutróficos ${ }^{7}$.

Pode-se observar que fatores de risco para DCV já estão presentes em crianças e adolescentes obesos, sendo o início e o tempo de duração da obesidade um fator importante no desenvolvimento da aterosclerose. Deve-se ressaltar que o ritmo de progressão do desenvolvimento do processo aterosclerótico é variável, dependendo do grau de exposição a uma série de fatores de risco.

\section{Resistência à insulina como fator desencadeante do diabetes melito tipo 2 e das alterações metabólicas associadas}

A resistência à ação da insulina no tecido e os níveis elevados de insulina plasmática em jejum, alterações bastante freqüentes em indivíduos obesos, parecem ser os primeiros sinais para o desenvolvimento do diabetes melito tipo 2. Nos indivíduos obesos, parece que nos estágios iniciais da doença, devido à resistência à insulina, as células B pancreáticas aumentam a produção e a secreção de insulina como mecanismo compensatório, enquanto a tolerância à glicose permanece normal. Este estado permanece durante algum tempo, até que se observa um declínio na secreção de insulina e, conseqüentemente, uma diminuição da tolerância à glicose. Portanto, o aumento da produção endógena de glicose acontece no estágio tardio do desenvolvimento do diabetes melito tipo $2^{8}$.

Até alguns anos atrás, o diabetes tipo 2 era uma doença encontrada mais no adulto; no entanto, nos últimos anos tem se verificado um aumento da prevalência desta doença em crianças e adolescentes. Neste sentido, deve-se enfatizar que o diabetes tipo 2 tem contribuído com mais de $30 \%$ dos novos casos de diabetes, mostrando uma possível relação do aumento da prevalência de obesidade infantil com o desenvolvimento desta doença.

Um estudo multicêntrico em 55 crianças e 112 adolescentes obesos constatou a existência da diminuição da tolerância à glicose em $25 \%$ e $21 \%$, respectivamente, sendo que $4 \%$ dos adolescentes eram diabéticos tipo 2 . O índice de resistência à insulina foi um forte preditor para a diminuição da tolerância à glicose, confirmando que na infância, a resistência à insulina associada com a hiperinsulinemia são os fatores de risco mais importantes para o desenvolvimento da diminuição da tolerância à glicose em crianças obesas ${ }^{9}$. O processo do desenvolvimento do diabetes tipo 2 na infância parece evoluir de maneira mais rápida do que nos adultos 6 . 
A hiperinsulinemia é considerada um fator de risco independente para a doença cardiovascular, já que tem um papel importante no desenvolvimento de outros componentes da síndrome metabólica, como a dislipidemia, a hipertensão e a hiperurecemia. Bao et al. ${ }^{10}$ avaliaram a longo prazo os níveis de insulina plasmática em crianças (5 a 9 anos de idade) e em adultos jovens (17 a 23 anos) da população do Bogalusa Heart Study e observaram as conseqüências de níveis elevados em relação ao perfil lipídico e à pressão arterial. Após 8 anos de acompanhamento, verificou-se que a prevalência de obesidade foi de $72 \%$ nos indivíduos que apresentavam níveis de insulina persistentemente elevados e casos de hipertensão e dislipidemia foram de 2,5 a 3,0 vezes maiores, respectivamente, para estes indivíduos.

\section{Alterações do perfil lipídico}

Em condições normais, a insulina tem várias ações na regulação do metabolismo lipídico; no entanto, nos indivíduos obesos o mesmo não é observado, devido às freqüentes alterações que ocorrem na atuação de determinadas enzimas e no metabolismo lipídico, devido à resistência a insulina. As principais alterações do perfil lipídico são: níveis aumentados de VLDL-C, devido à maior produção de triglicérides pelo fígado e ao menor catabolismo; redução dos níveis e do tamanho de HDL-C, relacionada à diminuição da subfração HDL-C2 e ao maior catabolismo devido à maior concentração de triglicérides nestas partículas, e partículas de LDL-C menores e mais densas, mais ricas em apolipoproteína $\mathrm{B}^{11}$.

Um estudo realizado com 2433 indivíduos de 9 a 24 anos de idade, na Finlândia, mostrou que $28 \%$ a $33 \%$ daqueles que se encontravam no maior quartil quanto aos níveis plasmáticos de insulina apresentavam pelo menos duas alterações metabólicas (pressão arterial sistólica alterada, níveis elevados de triglicérides e níveis baixos de HDL-C), em comparação a $9 \%$ dos que se encontravam no menor quartil ${ }^{12}$. Recentemente,
Valle et al. ${ }^{13}$ verificaram que das 61 crianças obesas avaliadas, 17 (27,9\%) eram hiperinsulinêmicas e apresentavam níveis plasmáticos de triglicérides significativamente mais elevados e de apolipoproteína A-I menores do que as com níveis normais de insulina. Neste estudo, a insulina foi um fator preditor independente para triglicérides $(p=0,0004)$ e apolipoproteína A-I $(p=0,005)$, mesmo quando ajustada pela idade, IMC e relação circunferência cintura/quadril, mostrando que níveis elevados de insulina têm uma influência direta sobre o perfil lipídico já nesta faixa etária.

\section{Alteração da pressão arterial}

A hiperinsulinemia também tem um papel importante no desenvolvimento da hipertensão em indivíduos obesos, conforme demonstrou o estudo de Ferrannini et al. ${ }^{14}$, no qual indivíduos obesos hipertensos tinham uma redução da sensibilidade à insulina. Os mecanismos que podem explicar a relação da hiperinsulinemia com o desenvolvimento da hipertensão são: a reabsorção renal de sódio e água aumentada; a ativação do sistema nervoso simpático; a diminuição da atividade enzima $\mathrm{Na}^{+}-\mathrm{K}^{+}$-ATPase e aumento do acúmulo de cálcio celular e o estímulo de fatores de crescimento. Por outro lado, a maior liberação de ácidos graxos livres (AGL) na veia porta, verificada em indivíduos com obesidade abdominal, parece ter uma relação com a fisiopatologia da hipertensão. Em indivíduos eutróficos o aumento de $A G L$, provocado artificialmente, aumenta a sensibilidade vascular por meio do estímulo alfa adrenérgico. Segundo estudos realizados em ratos, este efeito pode ser maior com o aumento do fluxo de $A G L$ no sistema porta. Ao inibir a produção de óxido nítrico, os AGL podem aumentar a pressão arterial, não somente pelo aumento da vasoconstricção, mas também pela diminuição do reflexo de relaxamento vascular ${ }^{15}$.

Aproximadamente, $20 \%$ a $30 \%$ das crianças obesas têm pressão arterial elevada, sendo que as obesas têm um risco de 2,4 vezes maior do que as eutróficas ${ }^{16}$. Em um estudo com 
1865 indivíduos de 6 a 24 anos de idade, a insulina também apresentou forte correlação com a pressão arterial sistólica ${ }^{17}$.

O trabalho de Cruz et al. ${ }^{18}$ mostrou ser a etnia (negros) e a baixa sensibilidade à insulina fatores independentes para níveis mais elevados de pressão arterial sistólica. A relação inversa entre sensibilidade à insulina e pressão arterial sistólica já é evidente nas primeiras décadas de vida.

\section{Alterações trombogênicas}

O acúmulo de gordura na região abdominal e a hiperinsulinemia também estão associados com um perfil trombogênico e inflamatório. Concentrações aumentadas de fibrinogênio e do inibidor do ativador do plasminogênio 1 (PAI-1) têm sido relatadas em indivíduos com obesidade visceral, aumentando o risco de trombose nestes indivíduos. Embora exista evidência de que possa existir uma forte relação da hiperinsulinemia com concentrações elevadas do PAI-1, é possível que este mecanismo seja dependente do estado metabólico e da quantidade de gordura corporal. Níveis elevados de certos marcadores inflamatórios como a interleucina 6 (IL-6), o fator de necrose tumoral e a proteína $C$ reativa também estão associados à obesidade abdominal ${ }^{19}$.

A presença de parâmetros homeostáticos não desejáveis já pode ser observada em crianças e adolescentes obesos, sendo o excesso de gordura corporal e a gordura abdominal os principais determinantes de níveis elevados de PAI-1 e fibrinogênio. Sudi et al. ${ }^{20}$ avaliaram 20 meninos e 40 meninas obesos e verificaram que após um programa para perda de peso (3 semanas com dieta hipocalórica e atividade física), houve uma redução significativa da adiposidade, do acúmulo de gordura na região abdominal e dos parâmetros fibrinolíticos), sendo que os fatores que melhor explicaram a redução de PAI-1 foram os níveis iniciais de PAI-1 e a perda de peso. Pode-se concluir que o excesso de peso, a gordura corporal e a obesidade abdominal são fatores preditores para alterações do sistema fibrinolítico em indivíduos mais jovens.

Portanto, alterações metabólicas aterogênicas, trombogênicas e inflamatórias contribuem para que crianças e adolescentes obesos com obesidade abdominal apresentem maior risco para o desenvolvimento da doença coronariana na vida futura. A hiperinsulinemia pode contribuir de maneira indireta para as aletrações trombogênicas, já que o acúmulo de gordura abdominal parece ser o preditor mais importante.

\section{Hiperuricemia}

Níveis elevados de ácido úrico no indivíduo obeso podem estar relacionados à resistência à insulina. Esta associação é explicada em parte pelo fato de a hiperinsulinemia, possivelmente, diminuir a excreção renal de ácido úrico ${ }^{21}$. No entanto, alguns estudos têm mostrado que níveis elevados de triglicérides estão mais relacionados com a hiperuricemia, podendo ser devido a um aumento do requerimento de NADPH para a síntese de novo de ácidos graxos nos indivíduos obesos, aumentando a produção de ácido úrico ${ }^{22}$. De qualquer forma, segundo este último mecanismo, a insulina estaria participando de maneira indireta; já níveis elevados deste hormônio estão associados a níveis altos de triglicérides. Em crianças obesas, tem-se observado níveis mais altos de ácido úrico quando comparados às eutróficas ${ }^{13}$, podendo esta alteração metabólica já estar presente em idades mais precoces.

\section{Níveis elevados de leptina}

A leptina é um hormônio secretado pelos adipócitos e o produto do gene ob, tendo influência na redução da ingestão alimentar e no aumento do gasto energético por meio da sua ação no hipotálamo, sugerindo que, possivelmente, em indivíduos obesos, exista resistência à ação desse hormônio. 
Em crianças obesas, têm sido verificados níveis aumentados de leptina. Alguns estudos avaliaram a relação da leptina com a sensibilidade à insulina, e constataram que, independente da obesidade, da idade, do sexo, da relação circunferência cintura-quadril, houve associação inversa, isto é, quanto menor a sensibilidade à insulina, maior o nível de leptina ${ }^{23}$. Trabalhos realizados com programas de intervenção para obesidade mostraram que, após a perda de peso, a redução dos níveis de leptina foi diretamente relacionada com a redução dos níveis de insulina, demonstrando haver uma relação direta entre estas duas variáveis; no entanto, Sudi et al. ${ }^{24}$ observaram que o principal determinante para a variação dos níveis de leptina em crianças e adolescentes obesos é o IMC e não a insulina basal e o índice de resistência à insulina.

Portanto, é complicado afirmar que existe uma relação direta entre níveis plasmáticos de leptina e de insulina. É possível que exista uma relação indireta, já que indivíduos obesos apresentam níveis elevados desses dois hormônios, sendo necessários mais estudos para esclarecer se existe uma relação direta entre essas duas variáveis.

Na infância e adolescência há diferença dos níveis plasmáticos de leptina entre os sexos: nas meninas, os níveis de leptina aumentam progressivamente de acordo com a idade, com o ganho de peso e com gordura corporal, enquanto que nos meninos ocorre uma diminuição progressiva. Alguns autores colocam que essa diferença se torna mais evidente na fase pré-puberal, enquanto outros, nos estágios finais de maturação sexual. Provavelmente às diferenças hormonais, em que a testosterona tem uma correlação negativa com níveis de leptina, explicam tais diferenças ${ }^{25}$.

A relação positiva e significativa da leptina com variáveis da síndrome metabólica (níveis elevados de insulina, triglicérides, LDL-C, colesterol total e pressão arterial sistólica e níveis baixos de HDL-C) foi observada em um grande estudo com 1264 crianças chinesas ${ }^{26}$. Crianças com níveis mais elevados de leptina (maior que o percentil 75) apresentavam valores significativamente mais elevados de pressão arterial sistólica, triglicerídeos e insulina do que aquelas com níveis mais baixos de leptina. Este hormônio pode ser visto como um marcador da obesidade e das alterações metabólicas relacionadas. A leptina foi considerada, em um grande estudo prospectivo, como fator de risco independente para doença cardiovascular27.

\section{Associação da gordura abdominal com as alterações metabólicas}

Uma vez que a obesidade andróide (maior depósito de gordura na região abdominal) apresenta uma grande associação com as alterações metabólicas, é importante verificar a distribuição da gordura corporal e a sua relação na etiologia da hiperinsulinemia e dos outros componentes da síndrome metabólica. Alguns trabalhos demonstraram que os homens apresentam em média 20\% ou mais da gordura total como gordura visceral, enquanto que as mulheres, menos de 10\%. Este dado é relevante já que a gordura visceral (intra-abdominal) está mais associada com as complicações metabólicas do que a gordura subcutânea abdominal e periférica ${ }^{11}$.

É importante destacar que durante a fase da puberdade existe uma diferença entre os sexos quanto à distribuição da gordura corporal. Nos meninos, ocorre uma redistribuição do acúmulo de gordura das extremidades para o tronco e nas meninas há uma tendência a ocorrer o inverso, sendo que esta diferença ocorre pelas modificações nos níveis de estrogênio e testosterona durante a puberdade. No entanto, Moreno ${ }^{28}$ relata que a quantidade excessiva de gordura corporal em adolescentes é acompanhada pelo maior depósito de gordura na região abdominal em relação às extremidades, independente do sexo.

$\mathrm{Na}$ obesidade abdominal, a atividade lipolítica celular está aumentada, ocorrendo uma maior liberação dos AGL na veia porta, expondo o fígado a uma quantidade aumentada de AGL. 
Isto diminui a extração hepática de insulina, contribuindo mais para o quadro de hiperinsulinemia sistêmica. O AGL em excesso contribuem para uma maior produção hepática de glicose, pela gliconeogênese, além de aumentarem a liberação hepática de lipoproteínas ricas em triglicérides, as VLDL-C, e apolipoproteína B na circulação ${ }^{11}$.

O efeito da distribuição da gordura corporal sobre o perfil metabólico em crianças e adolescentes obesos, está mais relacionada com alterações da síndrome metabólica do que a gordura periférica. Entretanto, a maioria dos trabalhos não avaliou diretamente a gordura visceral (intra-abdominal), a qual tem maior relação com as alterações metabólicas. Os métodos mais freqüentemente utilizados para avaliação da gordura abdominal são a circunferência abdominal, a relação da circunferência cintura-quadril e dobras cutâneas.

Para a distinção entre a gordura subcutânea e a gordura visceral seria necessária a utilização de métodos mais sofisticados, como a ressonância magnética ou a tomografia computadorizada. Em um estudo, realizado com crianças obesas, verificou-se que a gordura visceral, avaliada por meio da ressonância magnética, apresentou uma correlação significativa com níveis não desejáveis dos triglicérides, do colesterol total e frações, da relação Colesterol total sobre o HDLC, da apolipoproteína B e da pressão arterial sistólica ${ }^{29}$. Contudo, na prática clínica, a utilização desses métodos é inviável, recomendando-se então a utilização de medidas antropométricas que, por meio de equações, podem determinar a gordura visceral e subcutânea ${ }^{30}$.

A circunferência abdominal, medida no ponto médio entre o último arco costal e a crista ilíaca, tem sido utilizada no atendimento ambulatorial para avaliar a deposição de gordura na região abdominal, já que esta variável isolada tem demonstrado uma melhor associação com as alterações metabólicas do que a relação circunferência cintura-quadril (RCQ). Em adolescentes, parece que a RCQ não é apropriada como medida antropométrica para avaliar a distribuição da gordura corporal, pois a largura pélvica sofre alterações rápidas durante a maturação sexual, podendo a RCQ estar mais relacionada a essa variação do que à distribuição da gordura corporal, propriamente dita. Entretanto, ainda não foram estabelecidos pontos de corte para circunferência abdominal que indiquem o risco da criança ou do adolescente desenvolver a doença cardiovascular na vida futura.

\section{O N CLUS Ã O}

Pode-se concluir que a obesidade na infância e adolescência é um importante fator de risco para o desenvolvimento das doenças cardiovasculares na vida futura. O aumento da insulina plasmática pode ser considerado um sinal de alerta para o desenvolvimento do diabetes melito tipo 2 e das outras alterações metabólicas relacionadas. Desta maneira, torna-se necessária a implementação de medidas intervencionistas e de prevenção no combate a este distúrbio nutricional em indivíduos mais jovens. Dentre os principais componentes de políticas de uma vida saudável em adolescentes, destacam-se a promoção do aumento da atividade física, a implantação de programas de exercício físico e o incentivo à aquisição de hábitos alimentares saudáveis.

\section{A GRADECIMENTOS}

Associação Fundo de Incentivo à Psicofarmacologia (AFIP); CEPID/Instituto do Sono/FAPESP-UNIFESP; Departamento de Pediatria e Centro de Estudos em Psicobiologia e Exercício da UNIFESP.

\section{REFERÊ NCIAS}

1. Wang Y, Monteiro C, Popkin BM. Trends of obesity and underweight in older children and adolescents in the United States, Brazil, China, and Russia. Am J Clin Nutr 2002; (75):971-7. 
2. Balaban G, Silva GAP. Prevalência de sobrepeso em crianças e adolescentes de uma escola da rede privada de Recife. J Pediatria 2001; 77(2):96-100.

3. Stamler J. Epidemic obesity in the United States. Arch Intern Med 1993; 153:1040-4.

4. Must A, Jacques PF, Dallal GE, Bajema CJ, Dietz WH. Long-term morbity and mortality of overweight adolescents: a follow-up of the Harvard Growth Study 1922 to 1935. N Engl J Med 1992; 327:1350-5.

5. Srinivasan SR, Bao W, Wattigney WA, Berenson GS. Adolescent overweights associated with adult overweight and related multiple cardiovascular risk factors: The Bogalusa Study. Metabolism 1996; 45:235-40.

6. Styne DM. Childhood and adolescent obesity. Prevalence and significance. Pediatr Clin North Am 2001; 48(4):823-53.

7. Freedman DS, Dietz WH, Srinivasan SR, Berenson GS. The relation of overweight to cardiovascular risk factors among children and adolescents: The Bogalusa Heart Study. Pediatrics 1999; 103(6): 1175-82.

8. Weyer C, Bogardus C, Mott DM, Pratley RE. The natural history of insulin secretory dysfunction and insulin resistance in the pathogenesis of type 2 diabetes mellitus. J Clin Invest 1999; 104(6): 787-94

9. Sinha R, Fisch G, Teague B, et al. Prevalence of impaired glucose tolerance among children and adolescents with marked obesity. N Engl J Med 2002; 346(11):802-10

10. Bao W, Srinivasan SR, Berenson GS. Persistent elevation of plasma insulin levels is associated with increased cardiovascular risk in children and young adults. Circulation 1996; 93:54-9.

11. Després JP, Lemieux I, Tchernof A, Coullard C, Pascot A, Lemieux S. Distribution et métabolisme des masses grasses. Diabetes Metab 2001; 27(2):209-14

12. Ronnemaa T, Knip M, Lautala P, Viikari J, Uhari M, Leino $A$, et al. Serum insulin and other cardiovascular risk indicators in children, adolescents and young adults. Ann Med 1991; 23:67-72

13. Valle M, Gascón F, Martos R, Ruz FJ, Bermudo F, Morales R, et al. Metabolic cardiovascular syndrome in obese prepubertal children: the role of high fasting insulin levels. Metabolism 2002; 51(4): 423-8.

14. Ferrannini E, Buzzigoli G, Bonadonna R, et al. Insulin resistance in essential hypertension. $\mathrm{N}$ Engl J Med 1987; 317(6):350-7.

15. Sheehan MT, Michael DJ. Metabolic complications of obesity. Med Clin North Am 2000; 84(2): 363-85.

16. Lauer RM, Burns TL, Clarke WR, et al. Childhood predictors of future blood pressure. Hypertension 1991; 18(2):174-81.

17. Raitakari OT, Porkka KVK, Ronnemaa T, Knip M, Uhari $\mathrm{M}$, Akerblom HK, et al. The role of insulin in clustering of serum lipids and blood pressure in children and adolescents. Diabetologia 1995; 38(9):1042-50.

18. Cruz ML, Bergman RN, Goran MI. Unique effect of visceral fat on insulin sensivity in obese Hispanic children with a family history of type 2 diabetes. Diabetes Care 2002; 25(9):1631.

19. Hak AE, Stehouwer CD, Bots ML, et al. Associations of $C$-reactive protein with measures of obesity, insulin resistance, and subclinical atherosclerosis in healthy, middle-aged women. Arterioscler Thromb Vasc Biol 1999; 19(8):1986-91.

20. Sudi KM, Gallistl S, Weinhandl G, Muntean W, Borkenstein $\mathrm{MH}$. Relationship between plasminogen activator inhibitor-1 antigen, leptin, and fat mass in obese children and adolescents. Metabolism 2000; 49(7):890-5.

21. Facchini F, Chen I, Hollenbeck CB, Reaven GM. Relationship between resistance to insulin-mediated glucose uptake urinary uric acid clearance, and plasma uric acid concentration. JAMA 1991; 266:3008-11.

22. Clausen JO, Borch-Johnsen K, Ibsen H, Pedersen $\mathrm{O}$. Analysis of the relationship between fasting serum uric acid and the insulin sensivity index in a 
population-based sample of 380 young healthy Caucasians. Eur J Endocrinol 1998; 138:63-9.

23. Zimmet $P$, Collins V, de Courten $M$, et al. Is there a relationship between leptin and insulin sensivity independent obesity? A population-based study in the Indian Ocean nation of Mauritius. Int J Obes 1998; 21(3):171-7.

24. Sudi K, Gallistl S, Trobinger M, Reiterer E, Payerl D, Aigner $\mathrm{R}$, et al. Insulin and insulin resistance index are not independent determinants for the variation in leptin in obese children and adolescents. J Pediatr Endocrinol Metab 2000; 13(7):923-32.

25. Garcia-Mayor RV, Andrade MA, Rios M, Lage M, Dieguez C, Casanueva FF. Serum leptin levels in normal children: relationship to age, gender, body mass index, pituitary-gonodal hormones, and pubertal stage. J Clin Endocrinol Metab 1997; 82(9):2849-55.

26. Chu NF, Wang DJ, Shieh SM, Rimm EB. Plasma leptin concentrations and obesity in relation to insulin resistance syndrome components among school children in Taiwan - The Tipei Children Heart study. Int J Obes 2000; 24(10):1265-71.
27. Wallace $A M$, Mcmahon AD, Packard CJ, Kelly A, Shepherd J, Gaw A, et al. Plasma leptin and the risk of cardiovascular disease in the West of Scotland coronary prevention study. Circulation 2001; 104(25):3052-6.

28. Moreno LA. Fat distribution in obese and nonobese children and adolescents. J Gastroenterol Nutr 1998; 27(2):176-80.

29. Owens S, Gutin B, Ferguson M, et al. Visceral adipose tissue and cardiovascular risk factors in obese children has been shown to be more highly correlated with cardiovascular. J Pediatr 1998; 133(1):41-5.

30. Goran MI, Gower BA, Treuth M, et al. Prediction of intra-abdominal and subcutaneous abdominal adipose tissue in healthy pre-pubertal children and subcutaneous abdominal adipose tissue SA with body composition and anthropometry in children. Int J Obes 1998; 22(6):549-58.

Recebido para publicação em 8 de novembro de 2002 e aceito em 18 de junho de 2003. 